\title{
A Survey on Simulation Sickness in Virtual Environments
}

\author{
Factors, Design and Best Practices \\ Ananth N. Ramaseri Chandra, Fatima El Jamiy, Hassan Reza \\ School of Electrical Engineering and \\ Computer Science \\ University of North Dakota \\ Grand Forks, ND 58202 \\ Email: ananthnag.ramaserich, hassan.reza@und.edu
}

\begin{abstract}
Virtual Reality $(\mathrm{VR})$ is an emerging technology with a broad range of application in training, entertainment, and business. To maximize the potentials of virtual reality as a medium, the unwelcome feeling of simulation sickness needs to be minimized. Even with advancements in VR, the usability concerns are barriers for a wide-spread acceptance. Several factors (hardware, software, human) play a part towards a pleasant VR experience. The reviewed scientific articles are mostly part of documents indexed in digital libraries. In this paper, we review the potential factors which cause simulation sickness and minimize the usability of virtual reality systems. We review the best practices from a developer's perspective and some of the safety measures a user must follow while using the VR systems from existing research. Even after following some of the guidelines and best practices VR environments do not guarantee a pleasant experience for users. Limited research in VR environments towards requirement specification, design and development for maximum usability and adaptability was the main motive for this work.
\end{abstract}

Index Terms—Virtual reality(VR), Virtual environment, Simulation sickness, Head mounted display(HMD), Usability, Design, Guidelines, User

\section{INTRODUCTION}

Creating and interacting with alternative realities holds a different set of capabilities, change the consequences of our actions, and turn up human imagination. Virtual Reality (VR) is one such technology which helps us accomplish the impossible [1]. Recent advances in VR displays have granted for the greater adaptability of the technology. According to a report from AR Insider, 2020 approximately one in five of the US consumers are expected to use VR just in the year 2020 [2]. With the rise adaptation of VR, a distinctive problem which users face is simulation sickness. It also referred to as cybersickness a possible consequence of the intense visual and motion cuing. Wider audience are believed to experience sickness in a virtual environment [3]. Many researchers have identified the factors causing sickness or its symptoms in the recent years.

Researchers in Human Computer Interaction (HCI) and organizations associated with VR have been thriving on improving the usability of virtual environments [1] [4].

In this paper, we review the factors contributing to simulation sickness in a virtual reality environment and the necessary guidelines to minimize the same. We start off in section 2, by introducing usability, its importance in a virtual reality system, and the issues impacting the usability in VR systems. Additionally, we have classified the issues based of the existing research. In section 3, We introduce the concepts of Simulation sickness, cause, theories explaining sickness, its history, and how simulation sickness is a usability issues for virtual reality systems. In section 4 , we investigate the multiple contributing factors for simulation sickness and their types. In section 5, we review some of the existing guidelines and best practices for minimizing simulation sickness in virtual environments that help users minimize simulation sickness and experience improved presence in virtual environments.

We conclude the paper by identifying the need for unified design pattern to develop virtual reality applications to maximize usability.

\section{USABILITY}

Usability or the level of user satisfaction is a quality attribute of any given system. It is a combination of quality components like learnability, efficiency, satisfaction, memorability, and errors [5]. It can be measured by usability testing [6]. Surveying users executing real tasks while using the system and analyzing their feedback is known as usability testing. It is achieved by hard and soft methods. In both methods, the time to learn about the system, ease of use, errors made during the system operation are detailed to examine the usability of the system [7] [8].

\subsection{Virtual reality}

Virtual reality (VR) technology visualizes content in a threedimensional (3-D) perceptual space with immersive inter- 
action by a combination of advanced software, real-time constraints, and a user presence [9]. In a VR system, immersion is a vital characteristic as the user becomes a part of the simulated content. Humans visualize and interpret the environment around in three dimensions (3D). It helps us adapt to the virtual world which seems like the natural world [10]. Designing virtual systems based on human factors is said to have a sense of presence. i.e., immersion [11] [12].

\subsection{Usability issues in VR}

Exposure to virtual reality environments has varied effects on the user. Advanced research studies have to address the substantial issues in effectively adapting to the VR environments [13]. Issues in a virtual environment are classified into safety, direct, indirect effects, and social effects based on their impact area [13] [14].

TABLE 1

Classification of effects caused by a VR environment [13] [15].

\begin{tabular}{|l|l|}
\hline Effect Type & Impact area \\
\hline Direct & $\begin{array}{l}\text { Directly impact the human body targeting any } \\
\text { individual tissue. }\end{array}$ \\
\hline Microscopic & $\begin{array}{l}\text { Eye strain due to the visual flicker and distor- } \\
\text { tions from the HMDs. }\end{array}$ \\
\hline Macroscopic & Injury or trauma \\
\hline Indirect & $\begin{array}{l}\text { The physiological consequence of over expo- } \\
\text { sure to a VR environment at a functional level. }\end{array}$ \\
\hline
\end{tabular}

In Table 1, we have charted the classified effects of a virtual reality system. Direct effects of virtual environments deal with the health and safety risks of the users involved. They also cause multiple levels of damage. Furthermore, the microscopic issues will not cause visible damage, but the macroscopic issues may lead to trauma or serious injury. Indirect effects include image distortions and visual cues [13].

Interaction in virtual reality system is dependent on human senses. It is one of the main reasons for virtual reality developers to understand the physiology of human senses and emotions to build highly usable virtual systems. Even with a better understanding of the human physiology, a VR experience might be unrealistic if the optical flow patterns are not right or the rendered scene is lacking realism [16]. Users prone to light, motion, and sound sensitivity may have an uncomfortable virtual experience [14].

The understanding of the human ear and its ability to track audio sources also needs to be well understood. The intensity and frequency from the audio sources must be localized to help the user distinguish different sounds in the multidimensional audio.

Haptic touch, a mechanical stimulation that mimics the human touch enhances the usability and performance in VR. One known issue with haptic touch is that human skin adapts to it over some time and the sensitivity of the skin decreases.

Modern technologies involving human operations always needs to ensure health and safety. Social implications of the technology are less focused aspects of researcher. For example, the severity and violence in VR games is one of the concerns to be addressed. The virtual actions may be provocative in the real world. Lack of guidelines to gauge the violent behavior and difficulties in re-creating relationships outside laboratories are some of the issues in carrying out the research. Data available from lean existing research is insufficient to assume that VR provokes violent social behavior [13].

\section{Simulation Sickness}

Virtual reality systems provide an immersive experience with the help of high-resolution displays, spatial sound, and interactive devices. But such an interactive environment has its shortcomings which contribute to the physiological effects and post threats to the usability of the virtual reality environments.

Simulation sickness is a condition, which can result in headaches, problems standing up, straining eyes, disorientation, vertigo, loss of skin color, nausea, and vomiting. Though it is comparable to motion sickness, technologically it is different. It can appear while using a simulator or virtual reality devices. Simulation sickness is an area of research which includes many fields. All the existing research is from many years ago and they do not denote or consider the advancements in virtual reality and other simulation technologies [17]. Similar kind of sickness is induced when using VR system. Virtual reality induced symptoms and effects (VRISE) [18], Visually Induced Motion Sickness (VIMS), or Cybersickness are the terms used to address such sickness [4].

Cybersickness is a major issue that prevents the functional use of fully immersive virtual reality systems. It is a form of motion sickness and is one of the major health and safety concerns of the virtual environments. It poses a severe threat to the usability and performance of a virtual reality system [19]. Some of the issues associated with virtual reality systems are assumed to be a result of poor system design includes the virtual scene and user control strategies, the technical deficiencies like image distortions, visual cues, and also individual susceptibility of the user [20]. In addition to designing some human factors that limit exposure directions human performance also contributes to the reduced acceptability of virtual reality systems.

\subsection{Cause of sickness - Theories}

The occurrence of simulation sickness is topic for research from the early 19th century. The earliest theories addressing simulation sickness were based on cerebral anemia (deprived blood supply to the brain) or cerebral hyperemia(congestion of blood in brain and spinal cord). Advances in the studies paced in post the world-war era. Vestibular theory, Sensory conflict theory are some of the earliest ones which focus on simulation sickness [21].

According to the sensory conflict theory [21], simulation sickness is the result of the difference between the information received by the vestibular and the visual systems in a virtual environment. There is a strong mismatch where the visual information specifies movement, whereas the vestibular system indicates that the user is stationary. It is because of that conflict between the sensory inputs, the user experiences simulation sickness. 
Postural instability theory states that extended periods of postural instability causes the motion sickness symptoms and the level of sickness is directly proportional to the postural instability duration [22].

Poison theory states that the nauseating feeling caused by simulation sickness is the response of the body to the mismatch of visual and vestibular information. The body assumes some kind of food poisoning and causes feeling to throw up or any similar sensation [23].

Out of all the available theories (including eye movement theory, subjective vertical mismatch theory, negative reinforcement model) describing the cause of motion sickness in virtual reality systems the most widely accepted theory is the sensory conflict theory [20].

\subsection{Cybersickness as a Usability Issue}

Virtual reality delivers a window of opportunity for visualizing reality in an altered three-dimensional world. It helps individuals understand and provide limitless experiences, but is sometimes a clutter and also unsafe. For maximum effectiveness, it requires sensory awareness. The interactions and visual cues in virtual environments need to be well designed like the real world for VR to be understood and adapted.

The extent to which the user feels present in the environment indicates the success of the environment. Usability issues will diminish the sense of presence in a virtual environment. Users will not be able to experience the reality of a virtual environment if they must struggle using the environment. D. Geszten et al. [24], suggest that presence and copresence should be considered goals when designing VR environments and should be examined when evaluating the usability of these environments.

\section{FACTORS CONTRIBUTING TO SIMULATION SICK- NESS}

Many potential factors contribute to the simulation sickness in virtual environments. Such factors include age, gender, calibration, experience, and types of application. P.J Costello [25] in his work has tabulated many such factors based on individual, simulator type, and the task being performed. These factors have a profound impact on the severity of cybersickness. The factors affecting virtual reality have been grouped into the design, technicality of the VR system, environment in use, and participant types.

The Table. 2 below, provides an overview of some of the factors which are a concern for simulation sickness. In further sections of the paper, the factors are discussed in detail.

\subsection{Users}

\subsubsection{Age}

Simulation sickness is mostly based on sensory conflict theory and the postural instability theory. Literature suggests that younger people are more resistant to simulation sickness [17]. Vestibular perceptual threshold (weakest stimulus detected) is found to be diminishing in humans after the age of 40 making them more susceptible to simulation sickness
TABLE 2

Factors contributing towards Simulation Sickness

\begin{tabular}{|l|l|}
\hline Factors & Type \\
\hline \multirow{4}{*}{ User } & Age \\
\cline { 2 - 2 } & Gender \\
\cline { 2 - 2 } & Exposure to VR \\
\cline { 2 - 2 } & Control \\
\hline \multirow{4}{*}{ Display } & Head Mounted Displays \\
\cline { 2 - 2 } & Desktop displays \\
\cline { 2 - 2 } & Large curved displays \\
\hline \multirow{4}{*}{ VR Content type } & 3D Animations (Immersive) \\
\cline { 2 - 2 } & 360-degree videos (Semi immersive) \\
\cline { 2 - 2 } & Minimal or scenic (Non immersive) \\
\hline \multirow{3}{*}{ Design } & Interactions \\
\cline { 2 - 2 } & Realistic \\
\cline { 2 - 2 } & Sensory input \\
\hline
\end{tabular}

[26]. Era et al. have reported their findings that there are differences in postural balances between young and middleaged subjects who were part of their tests. Further at higher age groups the postural balance diminished [27].

\subsubsection{Gender}

Simulation sickness may vary according to gender with the use of HMD's. Schafer et al. [28] studied the effects of gender, technology, and their potential contribution towards simulation sickness. Using the data obtained from about 223 individuals (108 male and 115 women) they examined the levels of simulation sickness with regards to gender, sensory conflict, and improvements in VR technology. They conclude that women experienced a higher level of simulation sickness compared to men.

Stanney et al. [29] conducted multiple experiments and found that females were equally susceptible to motion sickness and it was due to the improper fit of the VR headset to Interpupillary distance (distance between the center of one's eyes). They also suggest a redesign to the VR headsets with alterable interpupillary distance to reduce the cybersickness in women.

\subsubsection{Exposure}

Stanney et al. [30] with their experiments concluded that with increase in exposure times is directly proportional to the severity of adverse symptoms. User groups vulnerable to motion sickness can experience approximately twice the level of effects when compared with non-susceptible individuals. Also, users enduring nauseant response during carnival rides can expect unpleasant symptoms. Brief exposure to virtual environments and dropping out while (or before) experiencing sickness and again retrying the same in a day or two will help the user adapt to the virtual environment. Recurrent exposures to the virtual environments are believed to lower or eliminate simulation sickness. But for that reason, it is not recommended using virtual environment for longer durations [17].

\subsubsection{Control}

User control and navigation also are strong contributing factors for simulation sickness as input devices (data glove, keyboard, mouse) can be used to control the virtual environment for interaction. Greater control over the environment may reduce the simulation. It will allow users to expect a 
reaction, after an action is performed [18]. It has been observed that navigating physically (like walking) has shown reduced symptoms than navigating through controllers [31].

\subsection{Displays}

A difference in environments like desktop VR and large screen curved displays or HMD's can have a varied impact on the level of sickness [18].

\subsubsection{Head Mounted Display (HMD)}

While working with head mounted displays factors like contrast, illumination, exposure duration, and working distance contribute towards straining the visual system. Using a stereoscopic HMD (EyePhone LX) in a immersive virtual environment for a ten minute duration, $60 \%$ of users reported symptoms like visual strain, nausea, and headache, while $20 \%$ reporting a reduction in binocular visual perception [32]. Similar symptoms were experienced by $61 \%$ of users after a twenty-minute exposure to immersive virtual content in a DVisor HMD [33]. Technical advancements in the VR display hardware (Oculus VR DK1 to Oculus VR DK2) did not have a significant impact towards the drop in the level of cybersickness [28].

Some symptoms may be more likely to occur in the virtual environments, Sensory conflict is a driving factor for causing nausea and other symptoms. While bodily and head movements-initiated disorientation, and incorrect optical design resulted in strain producing ocular symptoms. More recent contributions from Tong et al. [34] noticed that using HMD's caused a higher level of motion sickness when compared with the stereoscopic desktop displays. In this study, though some the users enjoyed the higher level of immersion in an HMD they could not sustain for longer periods.

\subsubsection{Large and Desktop Displays}

Former investigations resulted in reports stating that viewing time, distance (optimum viewing distance of $65 \mathrm{~cm}$ ) and lighting may also contribute to simulation sickness [35] [36]. Swindells et al. conclude that large displays by improving benefit sense of presence, but they do not directly impact or induce simulation sickness [37].

\subsection{VR Content Type}

\subsubsection{Immersion}

Guna et al. studied the impact of virtual content type on simulation sickness. They noticed that the type of video content (immersive or non-immersive) is a crucial factor for the usability of virtual environments. Video content type influenced the contributors' sensitivity to simulation sickness and their physiology. Their conclusion was based on the results of simulation sickness questionnaire, and other physiological measures. Lowest simulation sickness questionnaire score was recorded for non-immersive virtual content displayed on a television screen. While the highest scores were reported on an HMD with immersive content [38].

\subsubsection{Graphic realism}

Chang et al. in their work refer to the results of rendering realistic scenes. Participants who experienced realistic graphical content were prone to a higher level of simulation sickness. They also suspect that a sensory discrepancy in information between the vestibular and visual systems may be the cause for the higher level of discomfort. [39]

\subsubsection{Field of view}

Field of view (FOV) is the maximum visual angle of the virtual environment display. It is the visual range of the virtual world through the HMD or other display device. Altering the FOV of a display manually or dynamically, has reliably showed to reduce the discomfort caused in users during swift and rotating movements [39] [3].

\subsection{Design}

Virtual reality (VR) environments are among the emerging techniques for simulating real-world applications. Examples of successful application domains include training therapy and design. Despite the developments it is still hard to simulate realistic features to support a wide range of activities.

A persistent downside in designing and executing virtual environments is setting up the degree of similarity to the real-world, as human interactions with the real-world are highly associated to sensory information. Sustaining realism in the simulated world has its own benefits. Minimal training is needed for interacting when the simulated system is near to natural and is also correlated with usability of the system. In some cases which are far from real, there is a need for remarkable visual patterns based on the application environment.

Virtual reality is all about immersive environments which comprise of multi-modal (haptic, visual, and speech) inputs and outputs(HMD's and other displays) to provide maximum presence to the user. Designing complex virtual environments is an intriguing task as it requires managing hardware, general user safety, and the visual content which may cause disorientation or sickness. Though the design process for desktop VR environments is straightforward, the level of presence it draws is minimal and is less effective in tasks including physical interaction [40].

\section{EXISTING GUidelines to minimize SimULA- TION SICKNESS}

While hardware improvements are known to improve the usability of virtual environments [41], research also suggests some techniques to achieve maximum usability by minimizing the simulation sickness [42]. Also, Virtual reality hardware manufacturers like Oculus, HTC have detailed some design guidelines for content developers and hardware safety guidelines for users [43].

\subsection{Design}

While it is impossible to have an ideal set of guidelines, below mentioned are some of the important techniques to be considered for designing virtual environments inducing minimal symptoms of simulation sickness. 


\subsubsection{Latency and Frame rate}

Latency in VR is the delay between a user action and a visual reaction on a virtual environment display. Frame rate is a measure of how quick the frames pass through the rendering pipeline. A drop in frame rates is possible in a VR application with complex graphics. There is a high chance of experiencing simulation sickness if the latency between the user input and virtual content output is high [42].

While the minimum latency recommended is 20 milliseconds, anything higher than 46 milliseconds is known to induce motion sickness. For better quality virtual content Oculus, Sony, and Steam etc. have been emphasizing the importance of virtual content with low latency, responsiveness, and high frame rates [17] [44].

\subsubsection{Movement}

In a VR environment the movements of the character are not always made by the user. Such unavailability of movements can bring serious issues. Movements in a virtual environment should be realistic to match sensory expectations. Inappropriate movements like fast tilting, rolling, and movement in wave forms should be avoided. Some examples include gun sway, head bob, going up and down the stairs in a VR environment. Porcino et al. [42] suggest including movements based on jumps instead of continuous walks to minimize sickness. Uncontrolled user movement like flipping, falling or zoom transitions should be limited [17].

\subsubsection{Flicker}

Flicker is the brightness fluctuation on video displays and is known to cause sickness in a VR environment. It is visually disturbing and affects the health of the user's eyes. In larger displays the user is more likely to experience flicker in the edges of the screen, and in an HMD with a brighter screen, high refresh rate, it is essential to prevent flicker [17] [39].

\subsubsection{Rapid changes in acceleration and deceleration}

Rapid accelerating movements in virtual environments are a strong factor that promotes discomfort. Sensory conflicts that cause discrepancies in the brain occur because of sudden increased acceleration. It is also same with rapid deceleration. On the other hand, raising up the acceleration at a slower pace would result in pleasant experiences to the user [42]. In addition, rapid zoomed movements also should be avoided. The visual cones move faster than expected when a users' view is zoomed in [17].

\subsubsection{Sensory support}

A user might experience higher immersion in VR and expect relevant vestibular information after exposure to strong illusions. If the VR system cannot provide suitable sensory input, the system can cause motion sickness [3]. Designing a logical environment in which the players can focus on and bind to it. The elements of the user interface should be fixed rather than floating around. For instance, creating an environment with a clear steady horizon, reference points on which users can focus, rather than a world with imbalanced or changing background. In simple words, designing a virtual world which supports the human sensory systems is ideal for minimizing simulation sickness in a VR environment [17].

\subsubsection{Field of view (FOV)}

It is believed that a wide field of view (FOV) in a display can increase immersion in the virtual environment. Narrow FOV may affect the presence in the virtual environment which are some of the important characteristics. Displays with a wider FOV exhibit elevated occurrences of simulation sickness than with a narrow FOV [3]. With a narrow field of view, the amount of simulation in edges of the visual system is less which reduces the feeling of movement [42]. A FOV of 30 degrees has been suggested to largely minimize simulation sickness [45].

\subsubsection{Support shorter sessions of play}

Longer durations of exposure to virtual content can increase the discomfort levels. Application should be designed to support shorter durations of use to allow users to pause, rest, and then continue later. It would also be a better design if the application suggested users to take regular breaks, to avoid sickness [42]. Additionally, if a user is getting sick and leaves an application, there should be provisions for the user to come back and continue from the same stage in the application. Therefore, shorter sessions of exposure to the virtual content is considered beneficial [17] [39].

The Table 3. below, gives an overview of some of the design practices from the researchers and VR development organizations. While most of them are suggested part of their individual work, the same have not been collectively tested to obtain better usability results.

TABLE 3

Design guidelines for developers

\begin{tabular}{|l|l|}
\hline Guideline & Ideal \\
\hline Latency & $\begin{array}{l}\text { Low latency ( less than 20ms) [17] } \\
{[42]}\end{array}$ \\
\hline Frame rate & High [17] [44] \\
\hline Movement & $\begin{array}{l}\text { Realistic movements, Avoid invol- } \\
\text { untary [17] [42] }\end{array}$ \\
\hline Flicker & Avoid or minimize [39] \\
\hline Field of view FOV & Maximum of 30 degrees [45] \\
\hline $\begin{array}{l}\text { Rapid acceleration and de- } \\
\text { celeration }\end{array}$ & Avoid or minimize [17] [42] \\
\hline Sensory System & Design to support [3] \\
\hline Shorter sessions of play & Design to support [39] [42] \\
\hline
\end{tabular}

\subsection{User}

Organizations and developers have the ability of developing virtual content with minimal simulation sickness. Users can play their part in minimizing simulation sickness by following some best practices.

\subsubsection{Duration}

Using a virtual environment for longer durations improves the chances of simulation sickness. So, using them for a shorter duration, or taking breaks in between longer sessions may help in reducing the nauseating feeling during or after the exposure. 


\subsubsection{Focus}

In real life, human brains focus on a fixed point in space. VR confuses the brain as the fixed point in space looks farther, but the image is still on your headset screen with your eyes right next to it. Similarly, trying to focus on an object on the horizon in the VR scene may achieve reduced simulation sickness.

\subsubsection{Environment}

Simulation sickness symptoms strengthen in high temperature and poorly ventilated environments. Good air flow, ventilation in a room can help prevent nausea or support recovery after experiencing dizziness.

\subsubsection{Physical Health}

For an elevated level of presence, it is necessary for all the users' senses to be healthy. It is recommended being physically healthy and balanced to experience virtual reality at its best. If a user is suffering with cold, headaches or hangovers, it is better to avoid a virtual environment as the sickness symptoms may worsen.

The guidelines mentioned above are only to minimize the symptoms of simulation sickness and improve the usability of the VR systems. These do not rather help in eliminating the symptoms [17] [42].

\section{Conclusion}

Virtual reality has the potential to change entertainment, gaming, training, and many other industries. Regardless of the efforts from multiple research and development organizations, it is still not accepted by a wider audience due to the existing sickness and discomforts. This study is an extension of our earlier work which reasoned the usability and performance evaluation of virtual environments.

In this paper, we have examined the severity of cybersickness and found that the human factors, VR content and hardware trigger simulation sickness. In addition, the levels of cybersickness vary with the type of setup. We also have brought together some guidelines and best practices both for VR developers and users to minimize simulation sickness and improved experience in virtual environments. Yet, there is a need for a standardized development model to specify requirements, design and develop virtual reality applications which results in maximum adaptability.

\section{References}

[1] M. Al Zayer, Universal Usability of Virtual Reality. PhD thesis, 2019.

[2] A. Insider., "New report: 19 of u.s. adults have tried vr," May 2020.

[3] Y. Y. Kim, E. N. Kim, M. J. Park, K. S. Park, H. D. Ko, and H. T. Kim, "The application of biosignal feedback for reducing cybersickness from exposure to a virtual environment," Presence: Teleoperators and Virtual Environments, vol. 17, no. 1, pp. 1-16, 2008.

[4] N. McHugh, "Measuring and minimizing cybersickness in virtual reality," 2019.

[5] J. Nielsen, "Usability 101: Introduction to usability," Jan 2012.

[6] A. Anandhan, S. Dhandapani, H. Reza, and K. Namasivayam, "Web usability testing-care methodology," in Third International Conference on Information Technology: New Generations (ITNG'06), pp. 495-500, IEEE, 2006.

[7] C. Falcao and M. Soares, Applications of Haptic Devices \& Virtual Reality in Consumer Products Usability Evaluation, pp. 377-383. AHFE Conference, 012014.
[8] M. Galster and A. Widmer, "Quality attributes in medical planning and simulation systems," in Relating System Quality and Software Architecture, pp. 287-289, Elsevier, 2014.

[9] T. Mazuryk and M. Gervautz, "Virtual reality-history, applications, technology and future," 1996.

[10] F. El Jamiy and R. Marsh, "Survey on depth perception in head mounted displays: distance estimation in virtual reality, augmented reality, and mixed reality," IET Image Processing, vol. 13, no. 5, pp. 707-712, 2019.

[11] G. J. Kim, 3D Multimodal Interaction Design, pp. 122-152. London: Springer London, 2005.

[12] F. E. Jamiy and R. Marsh, "Distance estimation in virtual reality and augmented reality: A survey," in 2019 IEEE International Conference on Electro Information Technology (EIT), pp. 063-068, 2019.

[13] K. M. Stanney, R. R. Mourant, and R. S. Kennedy, "Human factors issues in virtual environments: A review of the literature," Presence, vol. 7, no. 4, pp. 327-351, 1998.

[14] E. Viirre and D. Bush, "Direct effects of virtual environments on users," in Handbook of Virtual Environments, pp. 581-588, CRC Press, 2002.

[15] A. N. Ramaseri Chandra, F. El Jamiy, and H. Reza, "A review on usability and performance evaluation in virtual reality systems," in 2019 International Conference on Computational Science and Computational Intelligence (CSCI), pp. 1107-1114, 2019.

[16] F. El Jamiy, A. N. Ramaseri Chandra, and R. Marsh, “Distance accuracy of real environments in virtual reality head-mounted displays," in 2020 IEEE International Conference on Electro Information Technology (EIT), pp. 281-287, 2020.

[17] B. Lewis-Evans, "Simulation sickness and vr - what is it, and what can developers and players do to reduce it?," Apr 2014.

[18] S. Sharples, S. Cobb, A. Moody, and J. R. Wilson, "Virtual reality induced symptoms and effects (vrise): Comparison of head mounted display (hmd), desktop and projection display systems," Displays, vol. 29, no. 2, pp. 58 - 69, 2008. Health and Safety Aspects of Visual Displays.

[19] P. Howarth and P. Costello, "Studies into the visual effects of immersion in virtual environments," Produced under HSE Research Contract, vol. 3181, p. R53, 1996.

[20] K. M. Stanney and R. S. Kennedy, Simulation sickness. ed Boca Raton, Florida: CRC Press, 2010.

[21] J. T. Reason and J. J. Brand, Motion sickness. Academic press, 1975.

[22] G. Riccio and T. Stoffregen, "An ecological theory of motion sickness and postural instability," Ecological Psychology - ECOL PSYCHOL, vol. 3, pp. 195-240, 091991.

[23] M. Treisman, "Motion sickness: An evolutionary hypothesis," Science (New York, N.Y.), vol. 197, pp. 493-5, 081977.

[24] D. Geszten, A. Komlódi, K. Hercegfi, B. Hámornik, A. Young, M. Köles, and W. G. Lutters, "A content-analysis approach for exploring usability problems in a collaborative virtual environment," 2018.

[25] P. J. Costello, "Health and safety issues associated with virtual reality: a review of current literature," 1997.

[26] M. Rey, T. Clark, W. Wang, T. Leeder, Y. Bian, and D. Merfeld, "Vestibular perceptual thresholds increase above the age of 40," Frontiers in Neurology, vol. 7, 102016.

[27] P. Era, P. Sainio, S. Koskinen, P. Haavisto, M. Vaara, and A. Aromaa, "Postural balance in a random sample of 7,979 subjects aged 30 years and over," Gerontology, vol. 52, pp. 204-13, 072006.

[28] D. M. Shafer, C. P. Carbonara, and M. F. Korpi, "Modern virtual reality technology: Cybersickness, sense of presence, and gender," Media Psychol Rev, vol. 11, 2017.

[29] K. Stanney, C. Fidopiastis, and L. Foster, "Virtual reality is sexist: But it does not have to be," Frontiers in Robotics and AI, vol. 7, p. 4, 2020.

[30] K. M. Stanney, K. S. Hale, I. Nahmens, and R. S. Kennedy, “What to expect from immersive virtual environment exposure: Influences of gender, body mass index, and past experience," Human Factors, vol. 45, no. 3, pp. 504-520, 2003. PMID: 14702999.

[31] D. Saredakis, A. Szpak, B. Birckhead, H. A. Keage, A. Rizzo, and T. Loetscher, "Factors associated with virtual reality sickness in head-mounted displays: a systematic review and meta-analysis," Frontiers in Human Neuroscience, vol. 14, 2020.

[32] M. Mon-Williams, J. P. Warm, and S. Rushton, "Binocular vision in a virtual world: visual deficits following the wearing of a headmounted display," Ophthalmic and Physiological Optics, vol. 13, no. 4, pp. 387-391, 1993. 
[33] E. Regan and K. Price, "The frequency of occurrence and severity of side-effects of immersion virtual reality.," Aviation, Space, and Environmental Medicine, 1994.

[34] X. Tong, D. Gromala, D. Gupta, and P. Squire, "Usability comparisons of head-mounted vs. stereoscopic desktop displays in a virtual reality environment with pain patients," vol. 220, 2016.

[35] S. S. Sasitorn Taptagaporn, "How display polarity and lighting conditions affect the pupil size of vdt operators," Ergonomics, vol. 33, no. 2, pp. 201-208, 1990.

[36] T. W. Dillon and H. H. Emurian, "Some factors affecting reports of visual fatigue resulting from use of a vdu," Computers in Human Behavior, vol. 12, no. 1, pp. 49 - 59, 1996.

[37] C. Swindells, B. A. Po, I. Hajshirmohammadi, B. Corrie, J. C. Dill, B. D. Fisher, and K. S. Booth, "Comparing cave, wall, and desktop displays for navigation and wayfinding in complex $3 \mathrm{~d}$ models," in Proceedings Computer Graphics International, 2004., pp. 420-427, 2004.

[38] J. Guna, G. Geršak, I. Humar, M. Krebl, M. Orel, H. Lu, and M. Pogačnik, "Virtual reality sickness and challenges behind different technology and content settings," Mobile Networks and Applications, pp. 1-10, 2019.

[39] E. Chang, H. T. Kim, and B. Yoo, "Virtual reality sickness: A review of causes and measurements," International Journal of Human-Computer Interaction, vol. 36, no. 17, pp. 1658-1682, 2020.

[40] M. B. Rosson and J. M. Carroll, Usability engineering : scenario-based development of human-computer interaction. 2002.

[41] K. Terzić and M. Hansard, "Methods for reducing visual discomfort in stereoscopic 3d: A review," Signal Processing: Image Communication, vol. 47, pp. $402-416,2016$.

[42] T. Porcino, E. Clua, C. Vasconcelos, D. Trevisan, and L. Valente, "Minimizing cyber sickness in head mounted display systems: design guidelines and applications," 112016.

[43] Oculus, "Introduction to best practices," 2020.

[44] K. Raaen and I. Kjellmo, "Measuring latency in virtual reality systems," pp. 457-462, 092015.

[45] W. Bles and A. Wertheim, "Appropriate use of virtual environments to minimise motion sickness," p. 10, 032001. 